\title{
Recent Progresses in the Growth of Two-dimensional Transition Metal Dichalcogenides
}

\author{
Yeonjoon Jung, ${ }^{\ddagger}$ Eunji Ji, ${ }^{\ddagger}$ Andrea Capasso, and Gwan-Hyoung Lee ${ }^{\dagger} \dagger$ \\ Department of Material Science and Engineering, Yonsei University, Seoul 03722, Korea \\ (Received January 7, 2019; Revised January 21, 2019; Accepted January 21, 2019)
}

\begin{abstract}
Recently, considerable progress and many breakthroughs have been achieved in the growth of two-dimensional materials, especially transition metal dichalcogenides (TMDCs), which attract significant attention owing to their unique properties originating from their atomically thin layered structure. Chemical vapor deposition (CVD) has shown great promise to fabricate large-scale and high-quality TMDC films with exceptional electronic and optical properties. However, the scalable growth of high-quality TMDCs by CVD is yet to meet industrial criteria. Therefore, growth mechanisms should be unveiled for a deeper understanding and further improvement of growth methods are required. This review summarizes the recent progress in the growth methods of TMDCs through CVD and other modified approaches to gain insights into the growth of large-scale and high-quality TMDCs.
\end{abstract}

Key words : Two-dimensional materials, Transition metal dichalcogenides, Growth, Chemical vapor deposition, Coalescence

\section{Introduction}

$\mathrm{T}$ wo-dimensional transition metal dichalcogenides (TMDCs) are a class of ultrathin semiconductors, which could serve as building blocks for next-generation technology. Atomically thin layers of these materials have been fabricated through simple mechanical exfoliation using a Scotch tape. This technique can produce crystalline and defect-free TMDC layers with excellent electronic properties; however, it is not scalable for industrial production. Therefore, many researchers have focused on the large-scale growth of $2 \mathrm{D}$ TMDCs using alternative techniques, such as chemical vapor deposition (CVD). CVD enables the growth of TMDCs in large-scale; however, issues related to the crystalline quality of the samples remain. Several contingencies, such as defects, grain boundaries (GBs), and wrinkles, can affect the TMDC properties. ${ }^{1)}$ Recently, many studies have been undertaken to control the many variables at play in CVD and optimize the quality of $2 \mathrm{D}$ TMDCs. The optimization strategies focused on different stages of the CVD process, such as nucleation, growth, and coalescence (formation of a continuous film), as shown in Fig. 1. For example, considering that the nucleation density determines the final film quality, the CVD parameters can be tuned to obtain a low number of nuclei that will ultimately merge into a continu-

\footnotetext{
"Contributed equally to this work

Corresponding author: Gwan-Hyoung Lee

E-mail : gwanlee@yonsei.ac.kr

Tel : +82-2-2123-7245 Fax : +82-2-2123-7245

ORCID

http://orcid.org/0000-0002-3028-867X
}

ous film made of a few single-crystal domains. ${ }^{2)}$ In general, control on the nucleation enables significant improvements in the surface coverage, layer thickness, and crystallinity. Other studies have focused on the GBs between adjacent TMDC grains, ${ }^{3)}$ because the presence of GBs in a polycrystalline film can significantly affect its electrical property. Control of GBs plays an important role also in view of industrial production, where material properties have to be predetermined and fully reproducible. ${ }^{4)}$

CVD is a promising technique for the growth of large-scale high-quality TMDC thin films. Various approaches have been used to deposit 2D TMDC on different kinds of substrates: (i) The evaporation of precursors in powder form is an effective means to supply the gas phase for growth (Fig. 2(a)). Usually, three different temperature zones are available to separately evaporate the metal source (such as $\mathrm{MoO}_{3}$ and $\mathrm{WO}_{3}$ ) and chalcogen source (such as $\mathrm{S}$ and Se) while the substrate is maintained at a different temperature. ${ }^{3,5)}$ For example, $\mathrm{MoO}_{3}$ vapor is decomposed to Mo to react with sulfur vapor, leading to the formation of $\mathrm{MoS}_{2}$ nuclei, which progressively expand into atomically thin crystals. (ii) Another approach is based on the reaction between a predeposited Mo or $\mathrm{MoO}_{3}$ thin film and sulfur precursor gas (Fig. 2(b)). ${ }^{6,7)}$ The $\mathrm{Mo}$ or $\mathrm{MoO}_{3}$ thin film with a thickness of a few tens of nanometers is deposited on the substrate by ebeam or thermal evaporation. The Mo surface usually becomes oxidized under ambient conditions during growth. This oxidized surface can be reduced by hydrogen gas. The $\mathrm{MoO}_{3}$ layer is, therefore, reduced to $\mathrm{MoO}_{2}$ or other reduced forms. After pre-annealing, prompt annealing at $1000^{\circ} \mathrm{C}$ in a sulfur gas atmosphere leads to the formation of a crystalline $\mathrm{MoS}_{2}$ thin film. (iii) In metal-organic chemical vapor 


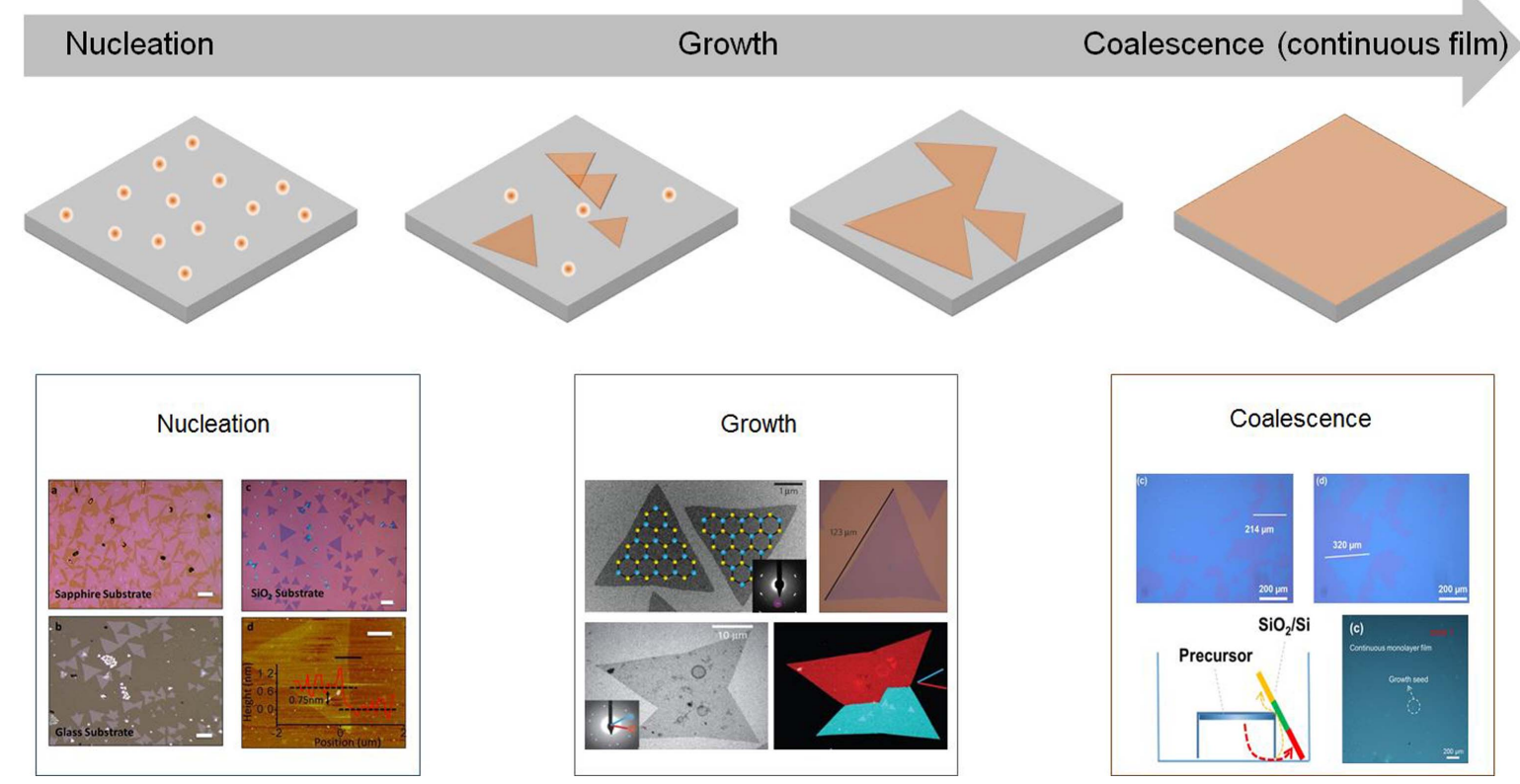

Fig. 1. Three different stages of the CVD process: nucleation, growth and coalescence (formation of continuous film).

\section{(a)}

\section{Evaporation of powder}
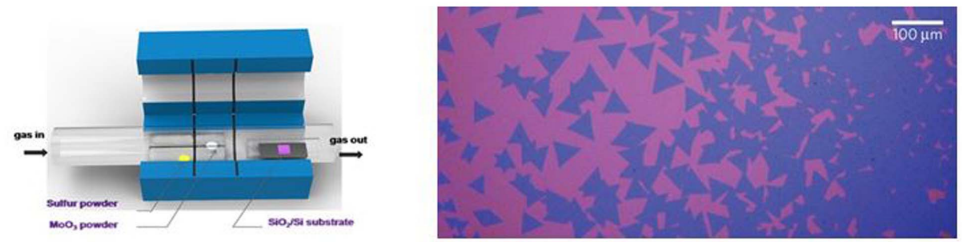

(b)

\section{Sulfurization of metal film}
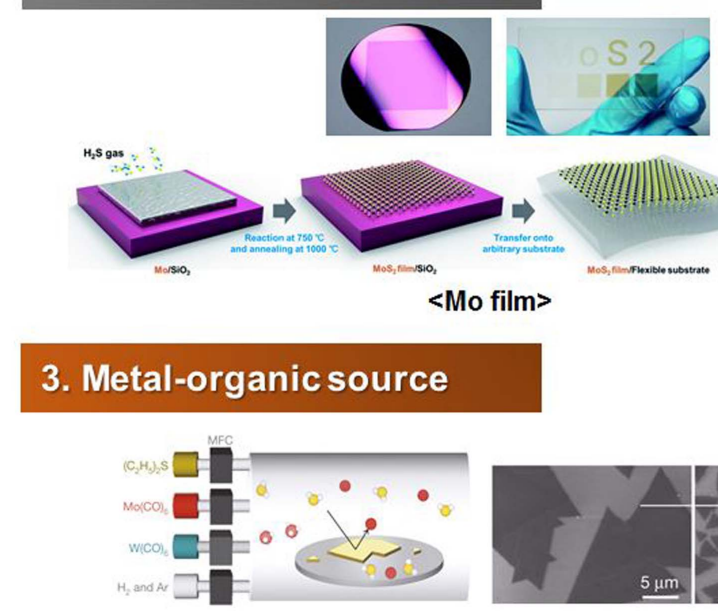
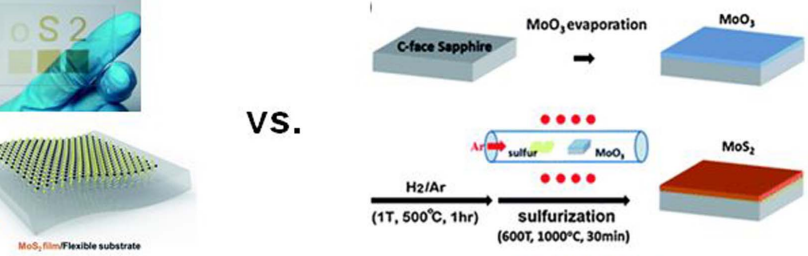

VS.

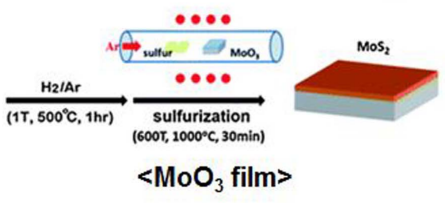

(c)
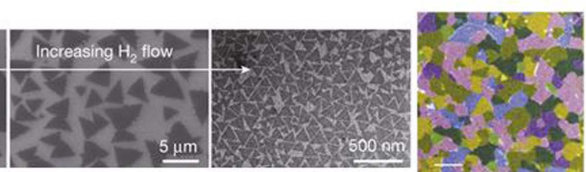

Fig. 2. Three representative CVD methods for growth of TMDCs. (a) Deposition of the evaporated powder precursors, (b) Chalcogenization (sulfurization/selenization) of pre-deposited $\mathrm{Mo}$ or $\mathrm{MoO}_{3}$ thin film, and (c) MOCVD with pyrolyzed metal organic gas precursors.

deposition (MOCVD), metal-organic gas precursors, such as $\mathrm{Mo}(\mathrm{CO})_{6}, \mathrm{~W}(\mathrm{CO})_{6}$, and $\left(\mathrm{C}_{2} \mathrm{H}_{5}\right)_{2} \mathrm{~S}$, are pyrolyzed on the target substrate for the growth of single-crystal or polycrystalline 2D TMDCs. Even though large-scale growth is possible at low temperatures, the required growth time might be over $12 \mathrm{~h}$, which is significantly longer than in the other approaches. Fig. 2(c) shows a sketch of the MOCVD growth process and optical images of $2 \mathrm{D} \mathrm{MoS}_{2}$ grown on a $\mathrm{Si} / \mathrm{SiO}_{2}$ 
substrate. ${ }^{8)}$ Because the gas phase precursors are generally diluted in a mixture of $\mathrm{Ar}$ and $\mathrm{H}_{2}$ carrier gases and the partial pressure of each reactant has to be precisely controlled throughout the process for large-scale growth, optimization of the process conditions in MOCVD is trickier.

\section{Nucleation}

\subsection{Homogeneous nucleation growth}

CVD is a widely employed technique that can be used to grow 2D TMDCs for various semiconductor applications. Among the various studies on the CVD of TMDCs, only a few researchers have focused on the details of the nucleation and growth mechanisms. Understanding these mechanisms will help scaling up the growth and facilitate an industrial production. Recently, Cain et $a .^{9)}$ elucidated the various stages of nucleation and growth of TMDCs. They directly grew single- and few-layer $\mathrm{MoS}_{2}$ and $\mathrm{MoSe}_{2}$ samples on ultrathin amorphous $\mathrm{SiO}_{2}$ membranes, as shown in Fig. 3(a). In their work, a partially sulfurized/selenized molybdenum trioxide $\left(\mathrm{MoO}_{3-\mathrm{x}}(\mathrm{S}, \mathrm{Se})_{\mathrm{y}}\right)$ core was fully covered with a fullerene-like shell of $\mathrm{MoS}_{2}-\mathrm{MoSe}_{2}$, as confirmed by aberration-corrected scanning transmission electron microscopy (STEM) imaging and energy-dispersive spectroscopy (EDS) mapping. As shown in Fig. 3(b), Zhu et al. ${ }^{10}$ carried out a transmission electron microscopy (TEM) study to reveal $\mathrm{MoS}_{2}$ growth kinetics when aided by nucleation seeds. They investigated the morphology, edge structure, and edge terminations at the atomic scale. The direct growth of few-layer and monolayer $\mathrm{MoS}_{2}$ onto graphenebased TEM grids aided direct TEM characterization without any transfer processes. Understanding these mechanisms can provide useful information to control the nucleation and growth of 2D TMDC materials.
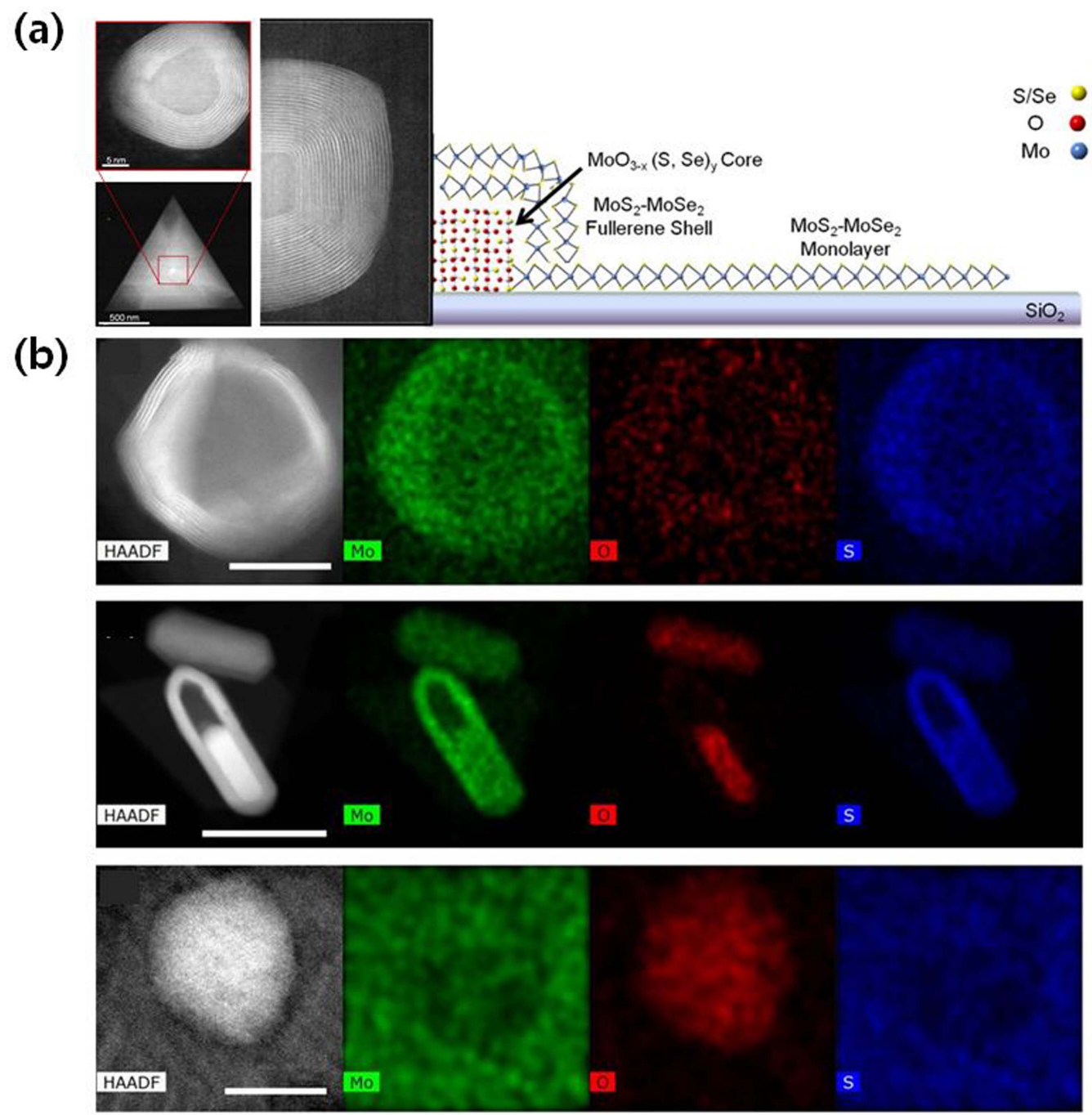

Fig. 3. (a) High-angle annular dark-field (HAADF) image of the nucleation center and schematic cross section structure of a nucleation center with the core-shell fullerene like structure. (b) Scanning transmission electron microscopy (STEM) - Xray energy dispersive spectroscopy (XEDS) mapping images of few-layer $\mathrm{MoS}_{2}$ structure, indicating the atomic structural and chemical composition (multi-shelled fullerene structure, multi-shelled tube, and a core without fullerene structure, respectively). (Scale bar: $20 \mathrm{~nm}$ ) 
(a)

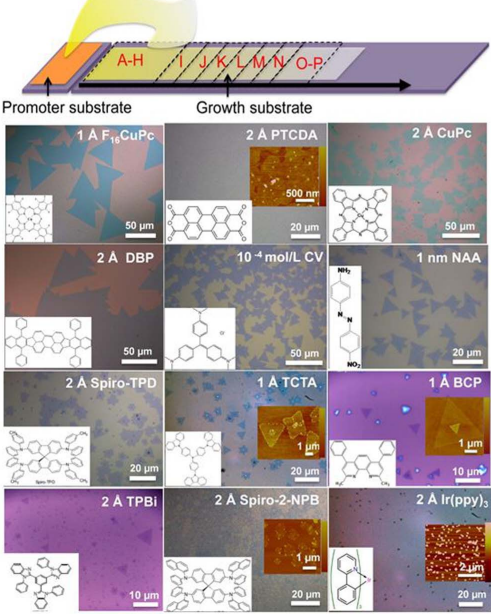

(b)

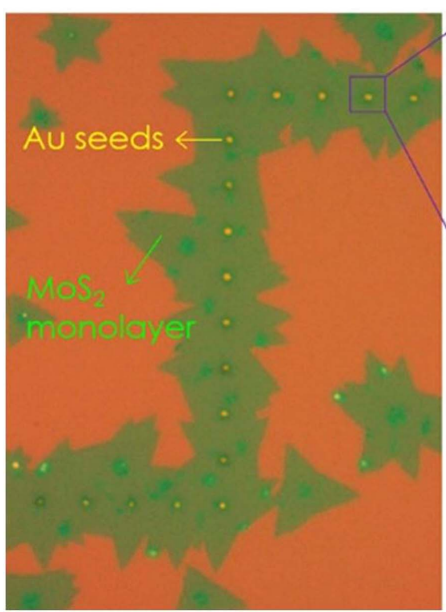

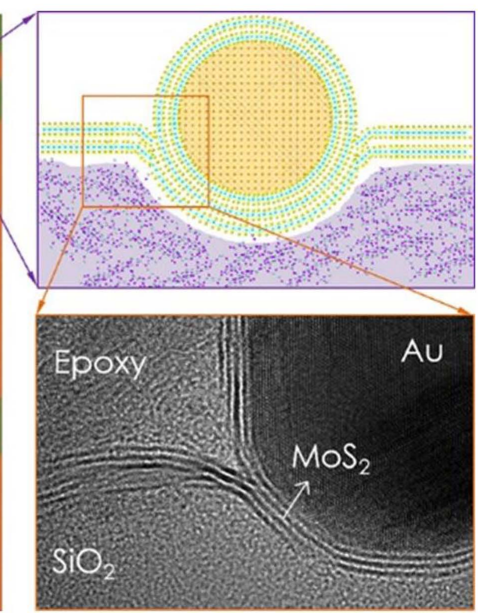

Fig. 4. (a) A schematic growth illustration of the promoter deposited substrate (top) and optical images of the surface after the $\mathrm{MoS}_{2}$ growth by different types of aromatic molecules as promoters (bottom). The insets show the corresponding molecular structures and atomic force microscopy (AFM) images of the surface after $\mathrm{MoS}_{2}$ growth. (b) Optical image of the MoS2 monolayers grown along $\mathrm{Au}$ seeds (left), and cross-section TEM image of the $\mathrm{MoS}_{2}$-encapsulated Au seed (right).

\subsection{Heterogeneous nucleation growth}

TMDCs are a class of materials with potential in various semiconductor applications. Many scientists have devoted their efforts to devise a production technique for monolayer TMDC, which would grant high yield and precise control on its properties, and ideally not require high-temperature processes $\left(<650^{\circ} \mathrm{C}\right)$. Although the conventional fabrication techniques still present several issues and hamper large- scale TMDCs, some progress has been made in lowering the required process temperatures using ammonium heptamolybdate $(\mathrm{AHM}),{ }^{11)}$ reduced graphene oxide, ${ }^{12)}$ or patterned $\mathrm{PT} / \mathrm{Ti} \operatorname{dots}^{13)}$ as seeding promoters on the $\mathrm{SiO}_{2} / \mathrm{Si}$ substrate. Conventionally, $\mathrm{MoS}_{2}$ island layers can be grown on various substrates without seeds $;{ }^{12)}$ however, the presence of seeding materials can favor the formation of initial nucleation sites that will foster the lateral growth of $\mathrm{MoS}_{2}$ crystals.

Table 1. Summary of growth results according to different seeding promoters

\begin{tabular}{|c|c|c|c|c|c|}
\hline & & & & Growth results & \\
\hline Types & $\begin{array}{l}\text { Seeding } \\
\text { promoter }\end{array}$ & $\begin{array}{c}\text { Sublimation/decomposition } \\
\text { temperature }{ }^{\mathrm{a}}\left({ }^{\circ} \mathrm{C}\right)\end{array}$ & $\begin{array}{l}\text { Domain size } \\
(\mu \mathrm{m})\end{array}$ & Thickness $^{\mathrm{b}}$ & Overall quality \\
\hline \multirow{13}{*}{ organic } & PTAS & $>600(\mathrm{~s})^{\mathrm{c}} /$ high $(\mathrm{d})$ & $\sim 60$ & $1 \mathrm{~L}$ & excellent \\
\hline & $\mathrm{F}_{16} \mathrm{CuPc}$ & $>430(\mathrm{~s})$ & $\sim 60$ & $1 \mathrm{~L}$ & excellent \\
\hline & PTCDA & $>450(\mathrm{~s})$ & continuous film & $1 \mathrm{~L}$ & good \\
\hline & $\mathrm{CuPc}$ & $>430(\mathrm{~s})$ & $\sim 30$ & $1 \mathrm{~L}$ & good \\
\hline & DBP & $350-450(\mathrm{~s})$ & $\sim 50$ & $1 \mathrm{~L}$ & good \\
\hline & $\mathrm{CV}$ & $>205(\mathrm{~d})$ & $\sim 20$ & $1 \mathrm{~L}$ & good \\
\hline & NAA & $>200(\mathrm{~d})$ & $\sim 15$ & $1 \mathrm{~L}$ & good \\
\hline & spiro-TPD & $>280(\mathrm{~s})$ & $\sim 10$ & $1 \mathrm{~L}$ & fair \\
\hline & TCTA & $>410(\mathrm{~s})$ & $\sim 10$ & $1 \mathrm{~L}$ and $\mathrm{ML}$ & fair \\
\hline & $\mathrm{BCP}$ & $>240(\mathrm{~s}) / 300(\mathrm{~d})$ & $\sim 5$ & $1 \mathrm{~L}$ and $\mathrm{ML}$ & fair \\
\hline & TPBI & $>350(\mathrm{~s})$ & $\sim 5$ & $1 \mathrm{~L}$ and $\mathrm{ML}$ & poor \\
\hline & spiro-2-NPB & $>390(\mathrm{~s})$ & $\sim 1$ & $1 \mathrm{~L}$ and $\mathrm{ML}$ & poor \\
\hline & $\operatorname{Ir}(\mathrm{ppy})_{3}$ & $>300(\mathrm{~s})$ & N/A & ML & bad \\
\hline \multirow{4}{*}{ inorganic } & $\mathrm{Al}_{2} \mathrm{O}_{3}$ & high (s\&d) & N/A & almost nothing & bad \\
\hline & $\mathrm{HfO}_{2}$ & high (s\&d) & N/A & almost nothing & bad \\
\hline & $5 \AA \mathrm{Au}$ & high (s\&d) & N/A & particles & bad \\
\hline & bare $\mathrm{Si}$ & high (s\&d) & N/A & almost nothing & bad \\
\hline
\end{tabular}

"The sublimation temperature is from thermogravimetry analysis (TGA). ${ }^{\mathrm{b}} 1 \mathrm{~L}$ : monolayer; ML: multilayer. ${ }^{\mathrm{c}} \mathrm{S}$ : sublimation; d: decomposition." 
Using perylene-3,4,9,10-tetracarboxylic acid tetrapotassium salt (PTAS) as a seeding promoter material, Ling et $a l .{ }^{14)}$ synthesized monolayer $\mathrm{MoS}_{2}$ at a low process temperature $\left(\sim 650^{\circ} \mathrm{C}\right)$, as shown in Fig. 4(a). Various aromatic molecules can also be used as effective seeding promoters to reduce the process temperature and increase the yield. The aromatic structure of these molecules is strongly related to the sublimation and decomposition temperatures. Table 1 reports the process conditions and properties of $\mathrm{MoS}_{2}$ films grown with different seeding promoters. Seeding promoters are an effective means to control growth; however, the position of the grown $\mathrm{MoS}_{2}$ crystal cannot be controlled and still remains random. Precise control of the crystal's position on the substrate is vital for the industry. Li et al. ${ }^{15)}$ used prepatterned metal (e.g., Au) nanoparticles as seeding materials to induce the formation of patterned geometries of monolayer $\mathrm{MoS}_{2}$ through CVD (Fig. 4(b)). In their work, $\mathrm{MoS}_{2}$ growth was lateral rather than vertical because of the more favorable thermodynamic driving force along the in-plane direction. ${ }^{16)}$ Studies of this kind can advance the design of patterned TMDC growth for future electronic industry.

\section{Control of Shape and Edge}

Unlike graphene, TMDCs are composed of two different elements; therefore, they have to be synthesized with two or more kinds of precursors. This adds to the complexity of the process, which might lower reproducibility. To solve this problem, several researchers studied in detail the effect of the concentration of precursors during TMDC growth. Using powder precursors, Wang et al. ${ }^{17)}$ experimentally (in Fig. 5(a)) demonstrated that the ratio of Mo and S affects the resultant shape and edge structure of $\mathrm{MoS}_{2}$ crystals, as shown in Fig. 5(b). When the ratio changes and the amount of molybdenum increases, triangular grains with only Mo-zz termination edge form. Conversely, when the sulfur ratio increases, triangular grains with only S-zz termination edge form. To explain this, Rajan et al. ${ }^{18)}$ performed theoretical calculations on the effect of the concentration ratio of two precursors, as shown in Fig. 5c. In their work, the optimal precursor concentrations were calculated to devise a systematic growth method with high reproducibility. Through proper control of the precursor ratio, a desired shape and edge structure was achieved, and hence the resulting effect on the TMDC properties was revealed. As shown in Fig. 5(d), Cao et al. ${ }^{19)}$ computationally analyzed the shape and edge structure of $\mathrm{MoS}_{2}$ single grains, and revealed magnetism characteristics. These series of calculations demonstrate the possibility of various catalytic activities as well as the synthesis of grains with the desired electronic and magnetic states by controlling the chemical potential through edge state control. ${ }^{20)}$

\section{Kinetics and Thermodynamics in Growth}

As shown in Fig. 6(a), the basic principle for thin-film growth is that the process proceeds in the most thermodynamically stable state. In this respect, it is very difficult to analyze the growth process in situ, because the process usually takes place in a sealed environment and at a high temperature. In addition, since the growth occurs in stages, it is too complicated to predict its evolution across the stages. In general, a kinetic and thermodynamic analysis of the process is the most advisable route to analyze and control the growth reaction. As shown in Fig. 6(b) and (c), Shang et $a l .{ }^{16)}$ proposed an analytical interpretation of the lateral and vertical growth of TMDCs by calculating the Gibbs free energy and investigating the phase diagrams of the various (a)

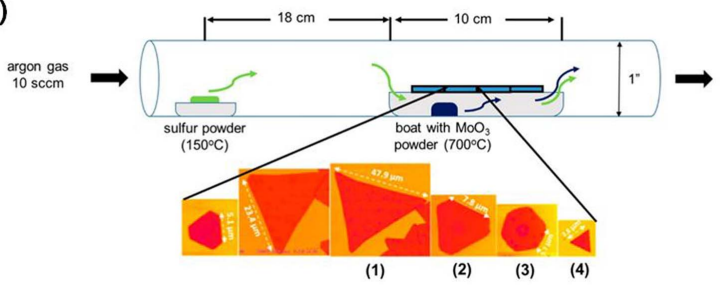

(b)

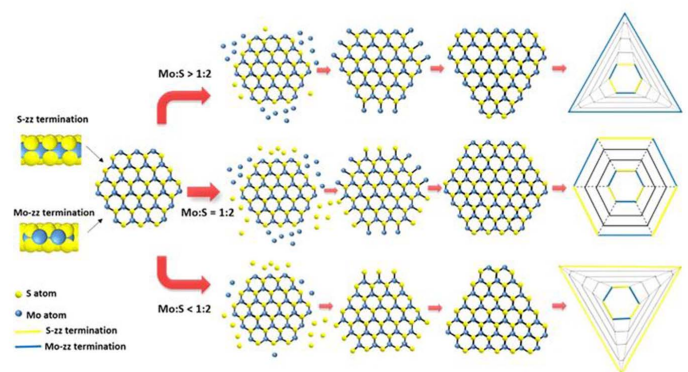

(c)

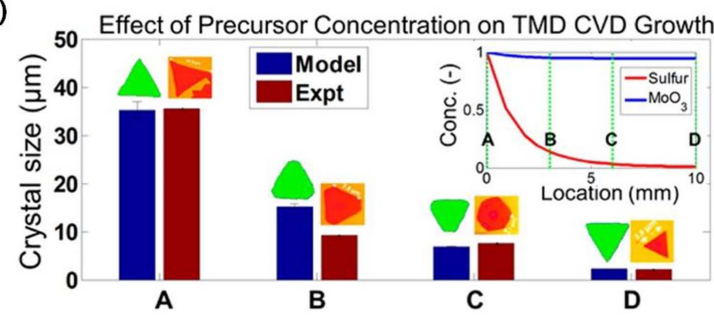

(d)

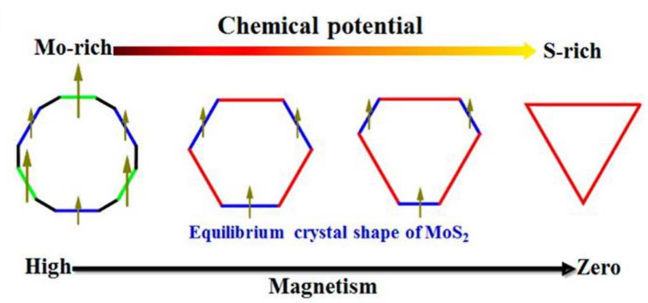

Fig. 5. (a) Schematic of the MoS2 growth by CVD and SEM images of the growth results. (b) Schematic illustration of the effect of the Mo : S atomic ratio on the grain shape. (c) Shapes and sizes of the MoS2 crystal as predicted by the model and SEM images of the growth results at different locations in the reactor. (d) The magnetic characteristic and morphology of MoS2 domains at different chemical potentials. 
(a)

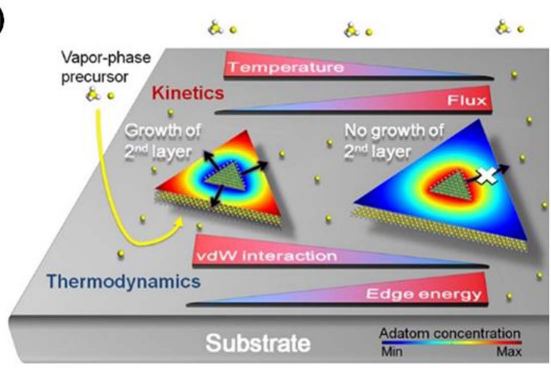

(b)

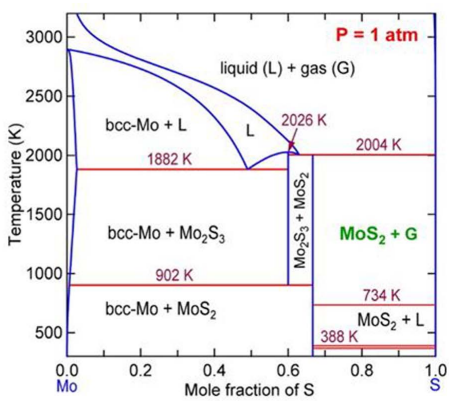

(d)

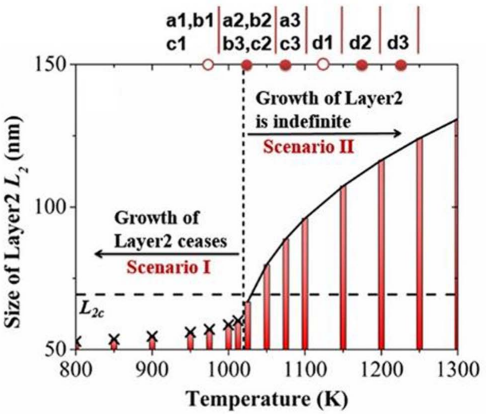

(c)

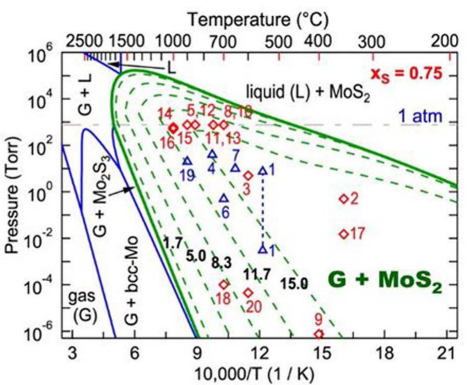

(e)

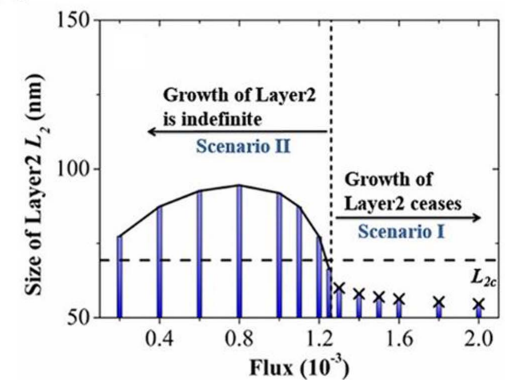

Fig. 6. (a) Profiles of adatom concentration on as-grown layer. (b) CALPHAD model of temperature-composition (T-x) Mo-S phase diagram at pressure $(\mathrm{P})=1 \mathrm{~atm}$. (c) CALPHAD model of P-T phase diagram of the Mo-S system under the S-rich condition (S : Mo $>2$, where $x_{s}=0.75$ ). The green dashed lines show that extra energy is added to $\mathrm{MoS}_{2}$. (d) Influence of temperature on the growth of newly growing layer (2nd layer). (e) Influence of flux (unit $\Omega \mathrm{s}$ ) on the growth of newly growing layer (2nd layer).

reactions occurring during $\mathrm{MoS}_{2}$ growth. Ye et al. ${ }^{21)}$ analyzed both the in-plane growth of a layer and the formation of vertically stacked layers in relation to the temperature and precursor mass flow rate, as shown in Fig. 6(d) and (e).
Their results suggest the possibility of qualitative and quantitative theoretical calculations that can estimate expected experimental results, thereby providing guidance for the design of effective growth processes. (a)

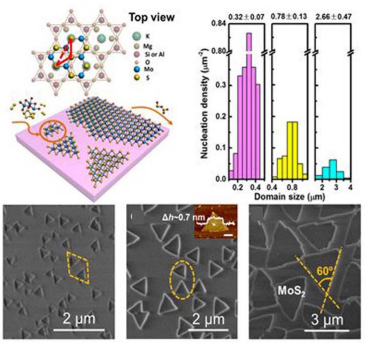

(c)

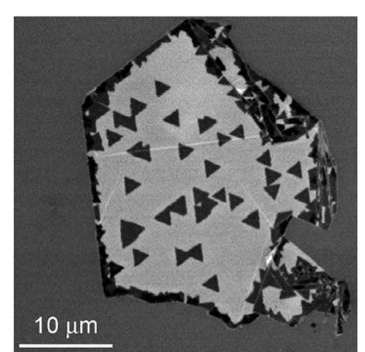

(b)

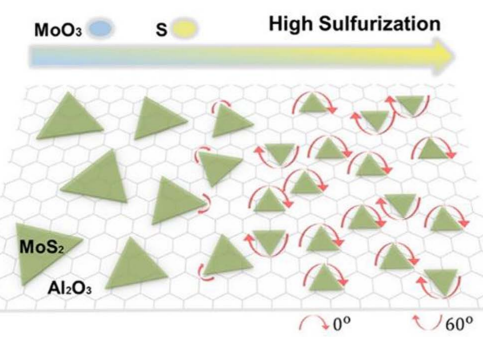

(d)

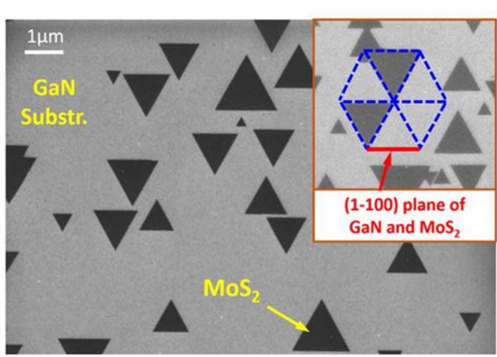

(e)

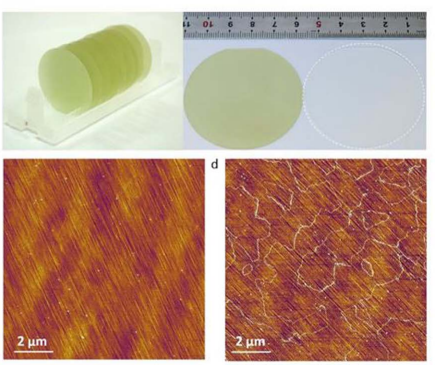

Fig. 7. (a) Statistical relationship between surface nucleation density and domain size from samples and SEM images of the initial growth of $\mathrm{MoS}_{2}$ on mica (Inset is the AFM profile of the $\mathrm{MoS}_{2}$ domain, scale bar $200 \mathrm{~nm}$ ). (b) Schematic illustration of $\mathrm{MoS}_{2}$ domains of two preferred orientations $\left(0^{\circ}\right.$ or $\left.60^{\circ}\right)$ registered on sapphire. (c) SEM image of triangular $\mathrm{WS}_{2}$ shapes grown on an h-BN flake. (d) SEM image of triangles of monolayer $\mathrm{MoS}_{2}$ on GaN epitaxial crystal. Alignment with (1-100) plane of $\mathrm{GaN}$ and scale bar : $1 \mu \mathrm{m}$ (Inset). (e) Photos of 2" $\mathrm{MoS}_{2} /$ sapphire and bare sapphire substrate and high-resolution AFM images of monolayer $\mathrm{MoS}_{2}$ on sapphire and $\mathrm{MoS}_{2}$ exposed in humid air (humidity : $55 \%$ ). 


\section{Epitaxial Growth}

As routinely used in the $\mathrm{Si}$ semiconductor industry, the epitaxial growth of TMDCs on single-crystal substrates is a viable approach. Several researchers have successfully grown different 2D TMDCs onto highly crystalline substrates, such as c-sapphire, ${ }^{22,23)}$ mica $^{24)}$ (Fig. 7(a)), GaN ${ }^{25)}$ (Fig. 7(d)), h-BN ${ }^{26)}$ (Fig. 7(c)), $\mathrm{SrTiO}_{3},{ }^{27)}$ and $\mathrm{MoS}_{2}{ }^{28)}$ where the grain orientation and crystallization can be finely controlled. Aljarb et al. ${ }^{22)}$ used annealed c-sapphire substrates for seed-assisted growth, and grew large-scale single-crystal $\mathrm{MoS}_{2}$ films. In their experiments, a highly sulfurized environment was shown to allow the efficient and aligned growth of $\mathrm{MoS}_{2}$ single crystals at a moderate temperature $\left(800^{\circ} \mathrm{C}\right)$, as shown in Fig. 7(b). Chen et al. ${ }^{28)}$ also performed highly organized homo-epitaxial growth using monolayer $\mathrm{MoS}_{2}$ as a substrate. Recently, Yu et al. ${ }^{23)}$ succeeded in synthesizing highly oriented wafer-scale $\mathrm{MoS}_{2}$ using a sapphire substrate. The synthesized $\mathrm{MoS}_{2}$ had a highly ordered orientation distribution ( $\sim 3^{\circ}$ difference) (Fig. 7(e)). This highly oriented $\mathrm{MoS}_{2}$ film could be easily peeled off from the sapphire substrate by simply immersing the substrate into water. In addition, Zhang et ll. $^{29)}$ used MOCVD to grow $\mathrm{WSe}_{2}$ grains aligned to the $0^{\circ}$ or $60^{\circ}$ directions of the sapphire substrate. These results show that growth on a substrate is key toward the growth of large-area, high-quality TMDCs.

\section{Assisted Growth}

Difficulties in optimizing the multi-factor CVD process for the growth of TMDCs have driven researchers to explore alternative approaches for the growth of high-quality TMDCs. In particular, promoters capable of inducing the hetero-nucleation of TMDC crystals have been targeted. Among them, the most popular promoter, PTAS, ${ }^{14)}$ has a molecular structure which enables TMDC growth without affecting the crystal properties. Similarly, as shown in Fig. 8(a), Choi et al. has succeeded in synthesizing high-quality $\mathrm{MoS}_{2}$ which suppresses sub-reaction by using $\mathrm{H}_{2} \mathrm{O}$ as a weak oxidizer during the MOCVD process. ${ }^{30}$ Also, many researchers created the hydroxyl form of metal oxide by supplying $\mathrm{H}_{2} \mathrm{O}$ molecules ${ }^{31-33)}$ for the efficient growth of $\mathrm{WS}_{2}$, which helped overcome the high thermal stability of metal oxide and more efficiently supplied metal atoms in a vapor state, leading to the growth of high-quality TMDCs. The biggest issue in conventional CVD growth using precursors in powder form is the chalcogenization of the metal source powder, which render it difficult to evaporate, causing a deficiency of metal atoms during the growth. As shown in Fig. 8(b), to prevent a shortage of the metal source, Chen et $a l{ }^{34)}$ maintained a uniform supply of the metal source vapor by oxidizing the metal source with a small amount of oxygen gas. Thus, they succeeded in synthesizing $\mathrm{MoS}_{2}$ single crystals with high quality and large sizes. As shown in Fig. 8(c), Zhou et al. ${ }^{35)}$ reported the growth of large, single-crystal $\mathrm{MoS}_{2}$ using an alkali metal halide. In their work, a metal oxychloride sub-reaction material was supplied instead of the conventional metal oxide vapor, resulting in the growth of a high-quality single-crystal $\mathrm{MoS}_{2}$ monolayer. Li et al. ${ }^{36,37)}$ also utilized alkali metal halide to synthesize various highquality TMDCs. As shown in Fig. 8(d), $\mathrm{MoS}_{2}$ nanoribbons with a width of tens to thousands of nanometers were fabricated through the vapor-liquid-solid (VLS) reaction. The (a)

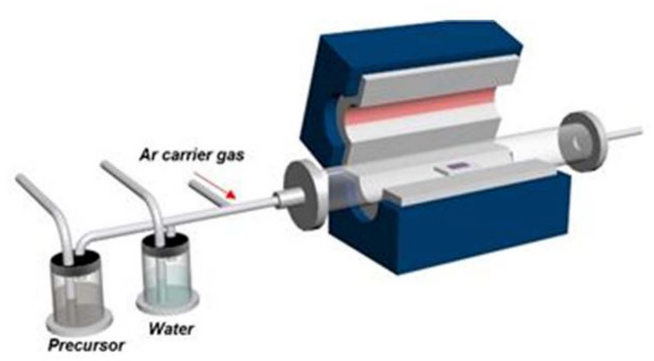

(c)

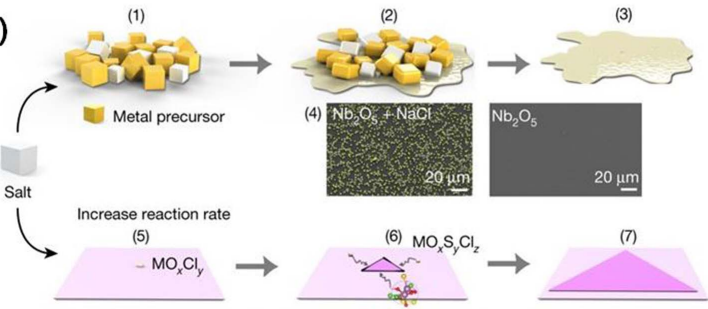

(b)
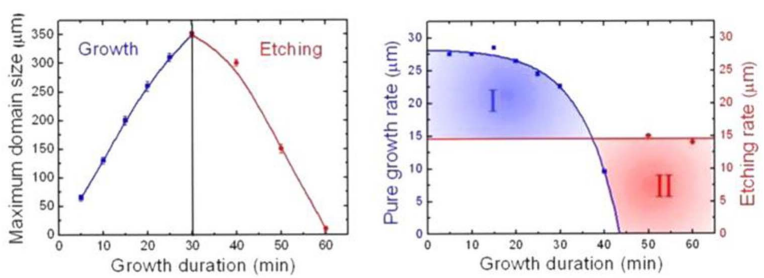

(d)
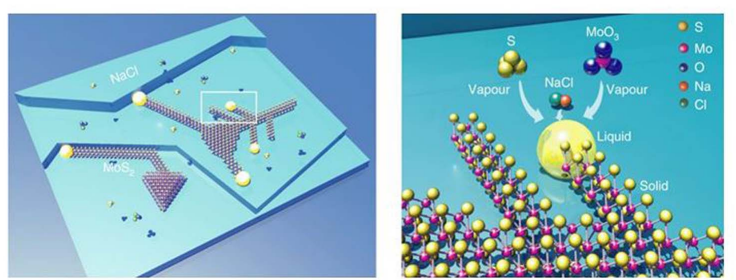

Fig. 8. (a) Schematic illustration of a CVD chamber connected to a liquid precursor and water bubbler. (b) Schematic illustration of the oxygen-assisted CVD process for $\mathrm{MoS}_{2}$ growth with sulfur and $\mathrm{MoO}_{3}$ powder. (c) Schematic illustration of the overall reactions and SEM images of the $\mathrm{Nb}$ nucleation with (left) and without (right) the aid of $\mathrm{NaCl}$ salts. (d) Schematic images of VLS synthesis of $\mathrm{MoS}_{2}$ ribbons on a NaCl crystal surface. 


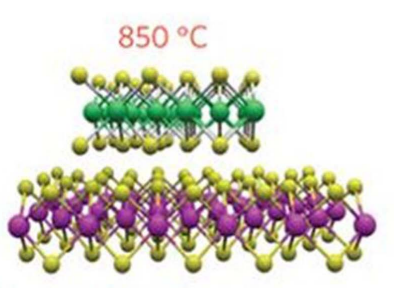

Vertical $\mathrm{WS}_{2} / \mathrm{MoS}_{2}$ bilayer

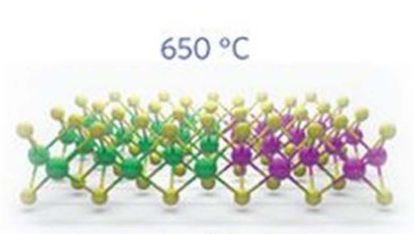

In-plane $\mathrm{WS}_{2} / \mathrm{MoS}_{2}$ monolayer
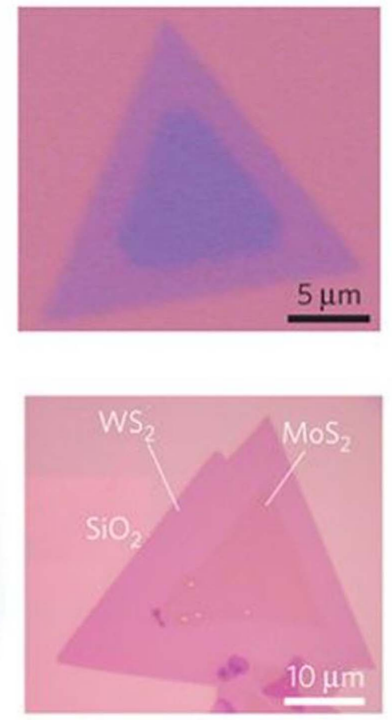
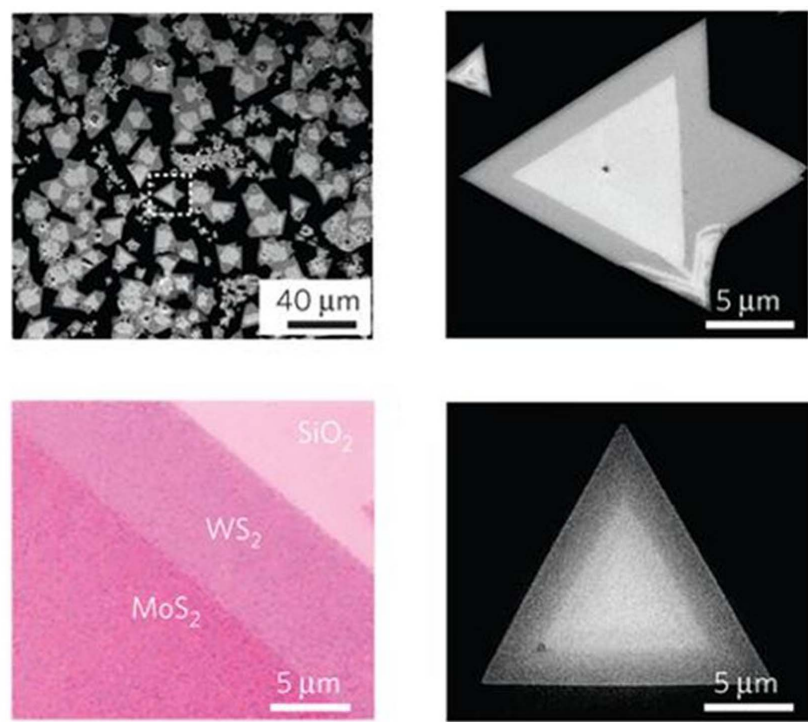

Fig. 9. (Top) Schematic, optical and SEM images of a vertical $\mathrm{WS}_{2} / \mathrm{MoS}_{2}$ heterostructure grown at $850^{\circ} \mathrm{C}$. (Bottom) Schematic, optical and SEM images of a lateral $\mathrm{WS}_{2} / \mathrm{MoS}_{2}$ heterojunction grown at $650^{\circ} \mathrm{C}$.

small amount of additives that act as nucleation seeds or growth promoters enables an improvement in the TMDC quality and in the growth control.

\section{Growth of Vertical and Lateral Heterostructures}

TMDCs have shown unprecedented properties in the monolayer limit. Hence, a combination of many TMDC monolayers, for example, vertical and lateral heterostruc- tures, can provide plenty of room to investigate novel properties at the junction of two (or more) different TMDCs. Even though multilayered heterostructures of TMDCs have been realized by transferring multiple layers, a more convenient and efficient way to fabricate large-scale and clean heterostructures is required. To overcome these drawbacks, many research groups have tried to directly fabricate assembled heterostructures during growth. As shown in Fig. 9, Gong et $a l .{ }^{38)}$ succeeded in synthesizing laterally (or vertically) stacked heterostructures by controlling the pro-

(a)

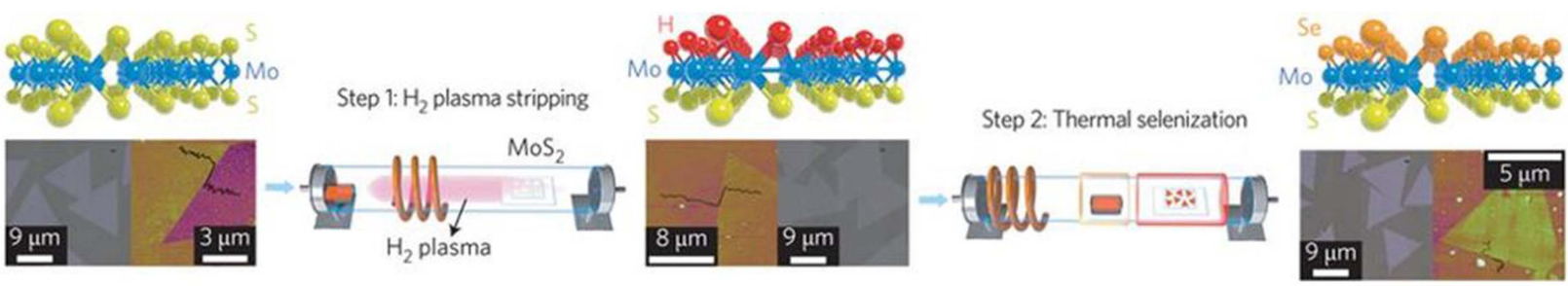

(b)

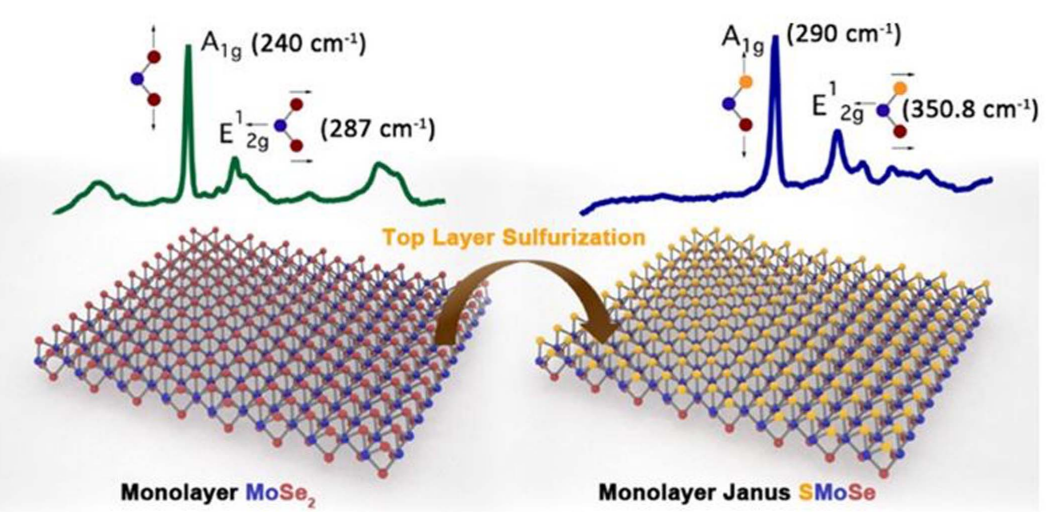

Fig. 10. (a) Schematic illustration of the CVD growth of Janus MoSSe monolayers, with optical and AFM images for each structure. (b) Sketch of the conversion process from MoSe2 to MoSSe, with Raman spectra of MoSe ${ }_{2}$ and Janus MoSSe, respectively. 
cess temperature in a two-step growth process. At relatively low process temperatures of $650^{\circ} \mathrm{C}$, the precursor did not overcome the activation energy required for vertical stacking of the additional layer on the basal plane of the previously grown TMDC layer. Therefore, the precursors bonded to a dangling bond at the edge site with a high surface energy, leading to the formation of an in-plane heterostructure. On the contrary, a high process temperature $\left(850^{\circ} \mathrm{C}\right)$ resulted in the growth of a vertically stacked heterostructure. Very recently, it was demonstrated that a Janus structure (in which two planes of different chalcogen atoms make the sandwich structure with the central metallic plane) can be synthesized, as shown in Fig. 10(a). ${ }^{39)}$ To fabricate the Janus plane of MoSSe, an intermediate state of MoSH, where the chalcogen atoms in the top $\mathrm{MoS}_{2}$ layer are substituted with $\mathrm{H}$ by hydrogen plasma, is prepared and then the Janus structure is formed by replacing hydrogen atoms with Se. Starting with $\mathrm{MoSe}_{2}$, Zhang et al. ${ }^{40)}$ also showed that the formation of the Janus structure is possible via direct sulfurization of the top surface of previously grown $\mathrm{MoSe}_{2}$, as shown in Fig. 10(b).

\section{Vertical and Helical Growth}

As shown in Fig. 11(a), Kong et $a .^{41)}$ successfully grew vertically aligned TMDC layers instead of the usually reported planar or nanoflower TMDC structures. Horizontally grow- ing TMDC layers have a limited catalytic effect owing to the absence of dangling bonds on the surface. However, during the growth of vertically aligned TMDCs, edge sites with a high surface energy (owing to dangling bonds) act as highly catalytic sites for the growth reactions. Therefore, the precise elucidation and control of these growth mechanisms is crucial for the growth of vertically aligned TMDCs. Occasionally, helically grown TMDCs were observed as reported by Zhang et al. (Fig. 11(b)). ${ }^{42)}$ Owing to the helical structure, the TMDCs showed optically active characteristics, which are in contrast to the properties of pyramid-shape multilayers. $^{43)}$

\section{Control of Layers and Stacking Order}

One of the unique characteristics of the TMDCs is that the band structure and band-gap size can be controlled by adjusting their thickness (i.e., the number of layers). However, there has been no clear way to control the thickness of TMDCs at the atomic layer scale in CVD because the monolayer is thermodynamically favorable. In TMDCs, there are no dangling bonds on the surface of the basal plane; however, dangling bonds are present at edge sites. Owing to the higher energy of edge sites (approximately 100 times higher than that of the basal plane), in-plane growth is particularly favorable. ${ }^{44)}$ Because of such stability of the basal plane, the precursor molecules deposited on the basal plane diffuse to (a)

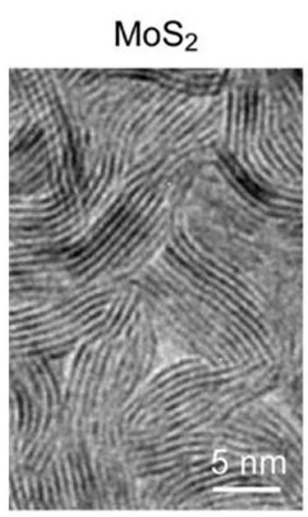

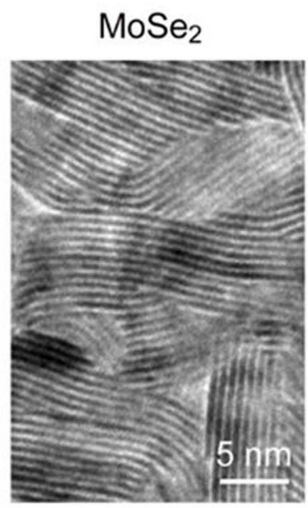

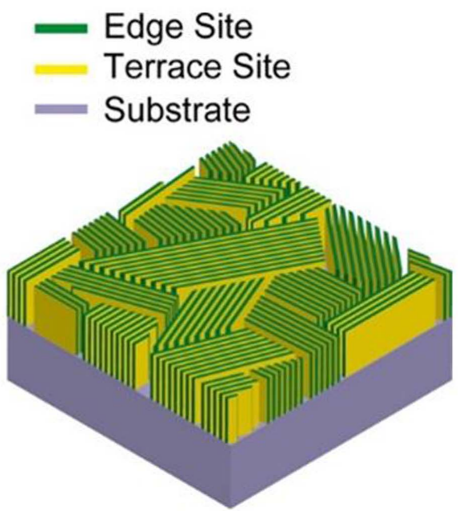

(b)

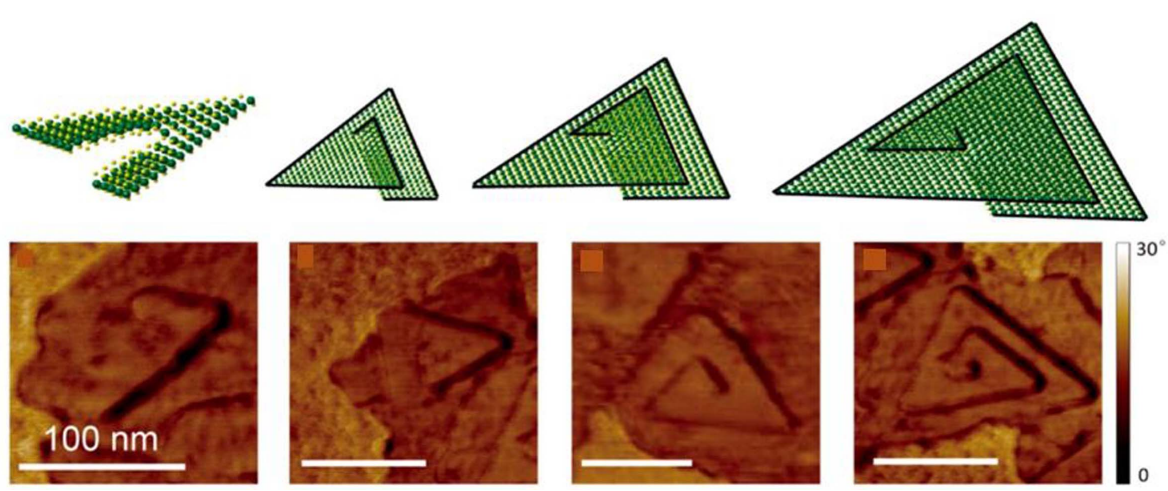

Fig. 11. (a) TEM images of $\mathrm{MoS}_{2}$ and $\mathrm{MoSe}_{2}$ film showing exposed edges, and schematic illustration of ideal edge-terminated structure of vertically grown molybdenum chalcogenide films. (b) Schematic image of the mechanism of spiral MoS structure (Top) and AFM images (phase image) of several spirals at different growth steps (bottom). 
(a)

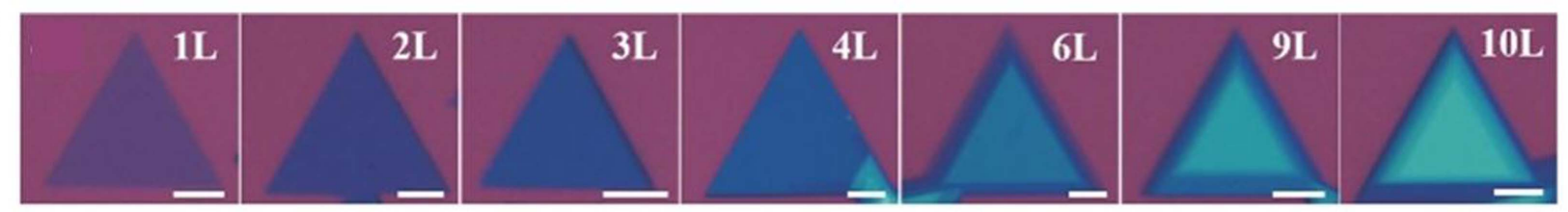

(b)
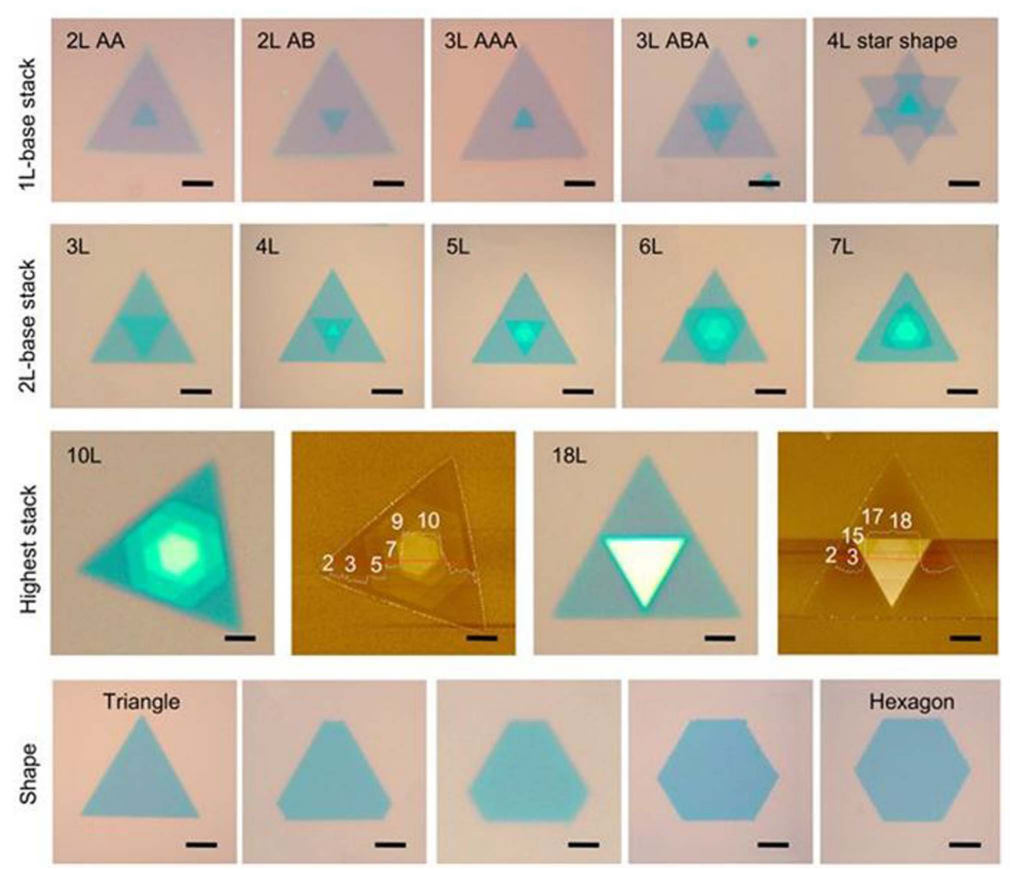

Fig. 12. (a) Optical microscope images of $\mathrm{MoS}_{2}$ flakes with different thicknesses from monolayer to ten layers (Scale bar: $5 \mu \mathrm{m}$ ). (b) Optical and AFM images for different layered stacking and shapes of $\mathrm{MoS}_{2}$ crystals (All scale bars: $5 \mu \mathrm{m}$ without bottom images; bottom images scale bar: $4 \mu \mathrm{m}$ ).

the active edge sites and form thick layers at the edge. ${ }^{45)}$ Therefore, thick TMDC crystals can be grown in the optimized growth condition. When the amount of the metal source is higher than that of the chalcogen source, layer-bylayer growth is more favorable than in-plane growth. As shown in Fig. 12(a), Zheng et $a l^{46)}$ successfully synthesized $\mathrm{MoS}_{2}$ with different thicknesses by CVD and electrochemically oxidized Mo films. High-quality $\mathrm{MoS}_{2}$ triangular single crystals were fabricated from a monolayer to ten layers by controlling the pressure of Mo from the pre-oxidized Mo film. Even in similar multilayers, the stacking order might be different. As shown in Fig. 12(b), Shinde et al. ${ }^{47)}$ synthesized various multilayers with different stacking types of $\mathrm{H}$ or $\mathrm{R}$ using $\mathrm{NaCl}$ as a promoter. Because multilayer TMDCs with different stacking sequences have distinct optical properties owing to the different interlayer coupling, control of the stacking order is critical for optical applications of multilayer TMDCs. Advanced MOCVD is a promising technique for the growth of multilayer TMDCs. However, MOCVD requires a long growth time for thick TMDCs owing to the very small amount of the supplied sources. Therefore, a new growth method, which enables the growth of multilayer TMDCs with controlled stacking order in a short time is needed for practical applications of TMDCs.

\section{Phase Control in Growth}

A distorted octahedral coordination leads to a metallic electronic structure, forming a 1T' phase, whereas a trigonal prismatic coordination forms a $2 \mathrm{H}$ phase, indicating semiconducting property. Molybdenum-based TMDCs are generally stable in the $2 \mathrm{H}$ phase under ambient conditions. In the monolayer form, the $2 \mathrm{H}$ phase of the semiconductor (which has a band gap of 1-2 eV) has no inversion symmetry, thereby leading to the generation of spin-split bands near the K-point of the Brillouin zone. In contrast, both $1 \mathrm{~T}^{\prime}$ and $1 \mathrm{~T}$ phases are metallic with inversion symmetry. Therefore, the phase transition of Mo-based TMDCs leads to variations in electrical properties, which can be utilized for various electronic applications. ${ }^{48-51)}$ Sung et $a l .{ }^{52)}$ synthesized metallic $\left(1 \mathrm{~T}^{\prime}\right)$ and semiconducting $(2 \mathrm{H}) \mathrm{MoTe}_{2}$ crystals within the same atomic plane by using hetero-epitaxial CVD with high-purity precursors $\mathrm{MoO}_{3}$ and Te and the promoter $\mathrm{NaCl}$. As shown in Fig. 13(a), to selectively stabilize each phase, the supply of $\mathrm{NaCl}$ and the process temperature were precisely controlled during the CVD. Empante et $a l .^{53)}$ fabricated three different $\mathrm{MoTe}_{2}$ phases, $1 \mathrm{~T}, 1 \mathrm{~T}$, and $2 \mathrm{H}$, by changing the growth quenching temperature (Fig. 13(b)). 
(a)

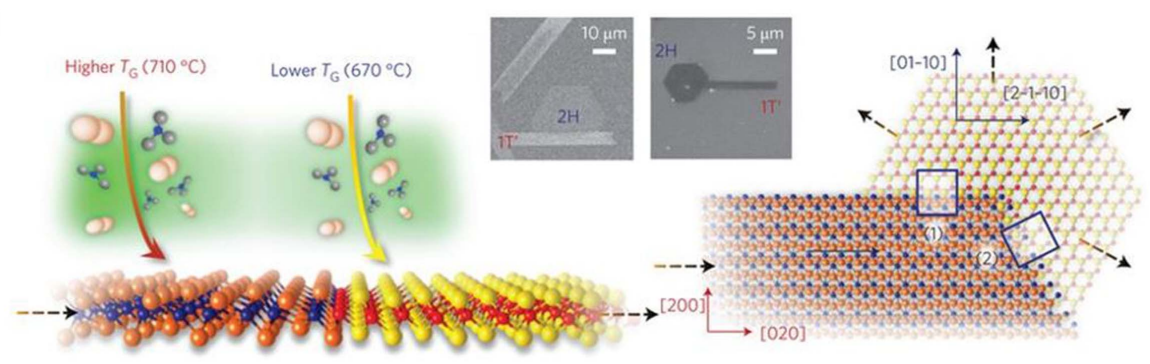

(b)

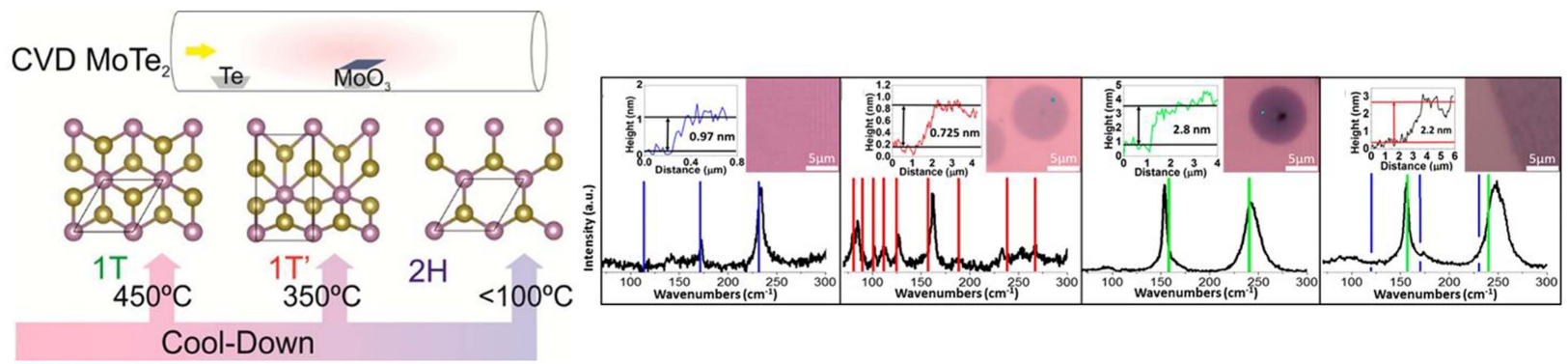

Fig. 13. (a) Growth scheme for coplanar hetero-epitaxy of $1 \mathrm{~T}^{\prime} / 2 \mathrm{H} \mathrm{MoTe}_{2}$ and SEM images of the crystallographic variants in the heterojunctions between $1 \mathrm{~T}^{\prime}$ - and $2 \mathrm{H}-\mathrm{MoTe}_{2}$ crystals. (b) Schematic illustration of different $\mathrm{MoTe}_{2}$ phases according to different cooling-down temperatures (Left); Raman spectra for each phase of $\mathrm{MoTe}_{2}$ with computational predications (colored lines), such as $2 \mathrm{H}$ continuous film, $1 \mathrm{~T}^{\prime}$ island, $1 \mathrm{~T}$ island, and mixed-phase film, respectively.

\section{Outlook}

In the beginning stage of TMDC growth, solid precursors in powder form are evaporated to supply precursor molecules. However, when solid precursors are used, it is not possible to maintain constant source concentrations and uniform deposition of the evaporated molecules. This limitation makes it difficult to utilize the current TMDC growth method for industrial purposes. To overcome this issue, modified CVD processes have been proposed. Recently, the control of phases and morphologies in the synthesis of TMDCs has received attention. Despite the discovery of growth mechanisms and their development, there are still many issues regarding the scalability, quality, reproducibility, and degradation. Nevertheless, the recent progress in the growth of TMDCs shows great promise to address existing issues.

\section{Acknowledgments}

This work was supported by the Korea Institute of Energy Technology Evaluation and Planning (KETEP) and the Ministry of Trade, Industry \& Energy (MOTIE) of the Republic of Korea (No. 20173010013340). This work was supported by the National Research Foundation of Korea Grant funded by the Korean Government (Grant No. 2017R1A5A1014862, SRC program: vdWMRC center). This research was supported by the National Research Foundation of Korea (NRF) grant funded by the Korean government (2018M3D1A1058794). A.C. is supported by the Yonsei University Research Fund (Yonsei Frontier Lab.
Young Researcher Supporting Program) of 2018.

\section{REFERENCES}

1. L. Tao, K. Chen, Z. Chen, W. Chen, X. Gui, H. Chen, X. Li, and J.-B. Xu, "Centimeter-Scale CVD Growth of Highly Crystalline Single-Layer $\mathrm{MoS}_{2}$ Film with Spatial Homogeneity and the Visualization of Grain Boundaries," ACS Appl. Mater. Interfaces, 9 [13] 12073-81 (2017).

2. S. Wu, C. Huang, G. Aivazian, J. S. Ross, D. H. Cobden, and X. Xu, "Vapor-Solid Growth of High Optical Quality $\mathrm{MoS}_{2}$ Monolayers with Near-Unity Valley Polarization," ACS Nano, 7 [3] 2768-72 (2013).

3. A. M. Van Der Zande, P. Y. Huang, D. A. Chenet, T. C. Berkelbach, Y. You, G.-H. Lee, T. F. Heinz, D. R. Reichman, D. A. Muller, and J. C. Hone, "Grains and Grain Boundaries in Highly Crystalline Monolayer Molybdenum Disulphide," Nat. Mater., 12 554-61 (2013).

4. Z. Lin, Y. Zhao, C. Zhou, R. Zhong, X. Wang, Y. H. Tsang, and Y. Chai, "Controllable Growth of Large-Size Crystalline $\mathrm{MoS}_{2}$ and Resist-Free Transfer Assisted with a $\mathrm{Cu}$ Thin Film," Sci. Rep., 518596 (2015).

5. J. Zhang, H. Yu, W. Chen, X. Tian, D. Liu, M. Cheng, G. Xie, W. Yang, R. Yang, and X. Bai, "Scalable Growth of High-Quality Polycrystalline $\mathrm{MoS}_{2}$ Monolayers on $\mathrm{SiO}_{2}$ with Tunable Grain Sizes," ACS Nano, 8 [6] 6024-30 (2014).

6. Y. Lee, J. Lee, H. Bark, I.-K. Oh, G. H. Ryu, Z. Lee, H. Kim, J. H. Cho, J.-H. Ahn, and C. Lee, "Synthesis of Wafer-Scale Uniform Molybdenum Disulfide Films with Control over the Layer Number Using a Gas Phase Sulfur Precursor," Nanoscale, 6 [5] 2821-26 (2014). 
7. Y.-C. Lin, W. Zhang, J.-K. Huang, K.-K. Liu, Y.-H. Lee, C.-T. Liang, C.-W. Chu, and L.-J. Li, "Wafer-Scale $\mathrm{MoS}_{2}$ Thin Layers Prepared by $\mathrm{MoO}_{3}$ Sulfurization," Nanoscale, 4 [20] 6637-41 (2012).

8. K. Kang, S. Xie, L. Huang, Y. Han, P. Y. Huang, K. F. Mak, C.-J. Kim, D. Muller, and J. Park, "High-Mobility Three-Atom-Thick Semiconducting Films with WaferScale Homogeneity," Nature, 520 656-60 (2015).

9. J. D. Cain, F. Shi, J. Wu, and V. P. Dravid, "Growth Mechanism of Transition Metal Dichalcogenide Monolayers: the Role of Self-Seeding Fullerene Nuclei," ACS Nano, 10 [5] 5440-45 (2016).

10. D. Zhu, H. Shu, F. Jiang, D. Lv, V. Asokan, O. Omar, J. Yuan, Z. Zhang, and C. Jin, "Capture the Growth Kinetics of CVD Growth of Two-Dimensional $\mathrm{MoS}_{2}$," npj $2 D$ Mater. Appl., 1 [1] 1-8 (2017).

11. G. H. Han, N. J. Kybert, C. H. Naylor, B. S. Lee, J. Ping, J. H. Park, J. Kang, S. Y. Lee, Y. H. Lee, and R. Agarwal, "Seeded Growth of Highly Crystalline Molybdenum Disulphide Monolayers at Controlled Locations," Nat. Commun., 6 [1] 6128 (2015).

12. Y. H. Lee, X. Q. Zhang, W. Zhang, M. T. Chang, C. T. Lin, K. D. Chang, Y. C. Yu, J. T. W. Wang, C. S. Chang, and L. J. Li, "Synthesis of Large-Area $\mathrm{MoS}_{2}$ Atomic Layers with Chemical Vapor Deposition," Adv. Mater., 24 [17] 232025 (2012).

13. Z. Wang, Q. Huang, P. Chen, S. Guo, X. Liu, X. Liang, and L. Wang, "Metal Induced Growth of Transition Metal Dichalcogenides at Controlled Locations," Sci. Rep., 6 38394 (2016).

14. X. Ling, Y.-H. Lee, Y. Lin, W. Fang, L. Yu, M. S. Dresselhaus, and J. Kong, "Role of the Seeding Promoter in $\mathrm{MoS}_{2}$ Growth by Chemical Vapor Deposition," Nano Lett., 14 [2] 464-72 (2014).

15. Y. Li, S. Hao, J. G. DiStefano, A. A. Murthy, E. D. Hanson, Y. Xu, C. Wolverton, X. Chen, and V. P. Dravid, "SiteSpecific Positioning and Patterning of $\mathrm{MoS}_{2}$ Monolayers: The Role of Au Seeding," ACS Nano, 12 [9] 8970-76 (2018).

16. S.-L. Shang, G. Lindwall, Y. Wang, J. M. Redwing, T. Anderson, and Z.-K. Liu, "Lateral Versus Vertical Growth of Two-Dimensional Layered Transition-Metal Dichalcogenides: Thermodynamic Insight into $\mathrm{MoS}_{2}$," Nano Lett., 16 [9] 5742-50 (2016)

17. S. Wang, Y. Rong, Y. Fan, M. Pacios, H. Bhaskaran, K. He, and J. H. Warner, "Shape Evolution of Monolayer $\mathrm{MoS}_{2}$ Crystals Grown by Chemical Vapor Deposition," Chem. Mater., 26 [22] 6371-79 (2014).

18. A. Govind Rajan, J. H. Warner, D. Blankschtein, and M. S. Strano, "Generalized Mechanistic Model for the Chemical Vapor Deposition of 2D Transition Metal Dichalcogenide Monolayers," ACS Nano, 10 [4] 4330-44 (2016).

19. D. Cao, T. Shen, P. Liang, X. Chen, and H. Shu, "Role of Chemical Potential in Flake Shape and Edge Properties of Monolayer $\mathrm{MoS}_{2}, "$ J. Phys. Chem. C, 119 [8] 4294-301 (2015).

20. M. Saab and P. Raybaud, "Tuning the Magnetic Properties of $\mathrm{MoS}_{2}$ Single Nanolayers by 3d Metals Edge Doping," J. Phys. Chem. C, 120 [19] 10691-97 (2016).

21. H. Ye, J. Zhou, D. Er, C. C. Price, Z. Yu, Y. Liu, J. Lowen- grub, J. Lou, Z. Liu, and V. B. Shenoy, "Toward a Mechanistic Understanding of Vertical Growth of van der Waals Stacked 2D Materials: A Multiscale Model and Experiments," ACS Nano, 11 [12] 12780-88 (2017).

22. A. Aljarb, Z. Cao, H.-L. Tang, J.-K. Huang, M. Li, W. Hu, L. Cavallo, and L.-J. Li, "Substrate Lattice-Guided Seed Formation Controls the Orientation of 2D TransitionMetal Dichalcogenides," ACS Nano, 11 [9] 9215-22 (2017).

23. H. Yu, M. Liao, W. Zhao, G. Liu, X. Zhou, Z. Wei, X. Xu, K. Liu, Z. Hu, and K. Deng, "Wafer-Scale Growth and Transfer of Highly-Oriented Monolayer $\mathrm{MoS}_{2}$ Continuous Films," ACS Nano, 11 [12] 12001-7 (2017).

24. Q. Ji, Y. Zhang, T. Gao, Y. Zhang, D. Ma, M. Liu, Y. Chen, X. Qiao, P.-H. Tan, and M. Kan, "Epitaxial Monolayer $\mathrm{MoS}_{2}$ on Mica with Novel Photoluminescence," Nano Lett., 13 [8] 3870-77 (2013).

25. D. Ruzmetov, K. Zhang, G. Stan, B. Kalanyan, G. R. Bhimanapati, S. M. Eichfeld, R. A. Burke, P. B. Shah, T. P. O'Regan, and F. J. Crowne, "Vertical 2D/3D Semiconductor Heterostructures based on Epitaxial Molybdenum Disulfide and Gallium Nitride," ACS Nano, 10 [3] 358088 (2016).

26. M. Okada, T. Sawazaki, K. Watanabe, T. Taniguch, H. Hibino, H. Shinohara, and R. Kitaura, "Direct Chemical Vapor Deposition Growth of $\mathrm{WS}_{2}$ Atomic Layers on Hexagonal Boron Nitride," ACS Nano, 8 [8] 8273-77 (2014).

27. Y. Zhang, Q. Ji, J. Wen, J. Li, C. Li, J. Shi, X. Zhou, K. Shi, H. Chen, and Y. Li, "Monolayer $\mathrm{MoS}_{2}$ Dendrites on a Symmetry-Disparate $\mathrm{SrTiO}_{3}$ (001) Substrate: Formation Mechanism and Interface Interaction," Adv. Funct. Mater., 26 [19] 3299-305 (2016).

28. J. Chen, X. Zhao, G. Grinblat, Z. Chen, S. J. Tan, W. Fu, Z. Ding, I. Abdelwahab, Y. Li, and D. Geng, "Homoepitaxial Growth of Large-Scale Highly Organized Transition Metal Dichalcogenide Patterns," Adv. Mater., 30 [4] 1704674 (2018).

29. X. Zhang, T. H. Choudhury, M. Chubarov, Y. Xiang, B. Jariwala, F. Zhang, N. Alem, G.-C. Wang, J. A. Robinson, and J. M. Redwing, "Diffusion-Controlled Epitaxy of Large Area Coalesced $\mathrm{WSe}_{2}$ Monolayers on Sapphire," Nano Lett., 18 [2] 1049-56 (2018).

30. S. H. Choi, B. Stephen, J. H. Park, J. S. Lee, S. M. Kim, W. Yang, and K. K. Kim, "Water-Assisted Synthesis of Molybdenum Disulfide Film with Single Organic Liquid Precursor," Sci. Rep., 7 [1] 1983 (2017).

31. T. Millner and J. Neugebauer, "Volatility of the Oxides of Tungsten and Molybdenum in the Presence of Water Vapour," Nature, 163 601-2 (1949).

32. G. Belton and A. Jordan, "The Volatilization of Molybdenum in the Presence of Water Vapor," J. Phys. Chem., 69 [6] 2065-71 (1965).

33. G. Belton and R. McCarron, "The Volatilization of Tungsten in the Presence of Water Vapor," J. Phys. Chem., 68 [7] 1852-56 (1964).

34. W. Chen, J. Zhao, J. Zhang, L. Gu, Z. Yang, X. Li, H. Yu, X. Zhu, R. Yang, and D. Shi, "Oxygen-Assisted Chemical Vapor Deposition Growth of Large Single-Crystal and High-Quality Monolayer $\mathrm{MoS}_{2}$," J. Am. Chem. Soc., 137 [50] 15632-35 (2015). 
35. J. Zhou, J. Lin, X. Huang, Y. Zhou, Y. Chen, J. Xia, H. Wang, Y. Xie, H. Yu, and J. Lei, "A Library of Atomically Thin Metal Chalcogenides," Nature, 556 355-59 (2018).

36. S. Li, Y.-C. Lin, W. Zhao, J. Wu, Z. Wang, Z. Hu, Y. Shen, D.-M. Tang, J. Wang, and Q. Zhang, "Vapour-LiquidSolid Growth of Monolayer $\mathrm{MoS}_{2}$ Nanoribbons," Nat. Mater., 17 535-42 (2018).

37. S. Li, S. Wang, D.-M. Tang, W. Zhao, H. Xu, L. Chu, Y. Bando, D. Golberg, and G. Eda, "Halide-Assisted Atmospheric Pressure Growth of Large $\mathrm{WSe}_{2}$ and $\mathrm{WS}_{2}$ Monolayer Crystals," Appl. Mater. Today, 1 [1] 60-6 (2015).

38. Y. Gong, J. Lin, X. Wang, G. Shi, S. Lei, Z. Lin, X. Zou, G. Ye, R. Vajtai, and B. I. Yakobson, "Vertical and In-Plane Heterostructures from $\mathrm{WS}_{2} / \mathrm{MoS}_{2}$ Monolayers," Nat. Mater., 13 1135-42 (2014).

39. A.-Y. Lu, H. Zhu, J. Xiao, C.-P. Chuu, Y. Han, M.-H. Chiu, C.-C. Cheng, C.-W. Yang, K.-H. Wei, and Y. Yang, "Janus Monolayers of Transition Metal Dichalcogenides," Nat. Nanotechnol., 12 744-49 (2017).

40. J. Zhang, S. Jia, I. Kholmanov, L. Dong, D. Er, W. Chen, H. Guo, Z. Jin, V. B. Shenoy, and L. Shi, "Janus Monolayer Transition-Metal Dichalcogenides," ACS Nano, 11 [8] 8192-98 (2017).

41. D. Kong, H. Wang, J. J. Cha, M. Pasta, K. J. Koski, J. Yao, and Y. Cui, "Synthesis of $\mathrm{MoS}_{2}$ and $\mathrm{MoSe}_{2}$ Films with Vertically Aligned Layers," Nano Lett., 13 [3] 1341-47 (2013).

42. L. Zhang, K. Liu, A. B. Wong, J. Kim, X. Hong, C. Liu, T. Cao, S. G. Louie, F. Wang, and P. Yang, "Three-Dimensional Spirals of Atomic Layered $\mathrm{MoS}_{2}$," Nano Lett., 14 [11] 6418-23 (2014).

43. J. Zhang, M. Ye, S. Bhandari, A. K. M. Muqri, F. Long, S. Bigham, Y. K. Yap, and J. Y. Suh, "Enhanced Second and Third Harmonic Generations of Vertical and Planar Spiral $\mathrm{MoS}_{2}$ Nanosheets," Nanotechnology, 28 [29] 295301 (2017).

44. J. Verble, T. Wietling, and P. Reed, "Rigid-Layer Lattice Vibrations and van der Waals Bonding in Hexagonal $\mathrm{MoS}_{2}$," Solid State Commun., 11 [8] 941-44 (1972).

45. D. Kong, W. Dang, J. J. Cha, H. Li, S. Meister, H. Peng, and Z. Liu, and Y. Cui, "Few-Layer Nanoplates of $\mathrm{Bi}_{2} \mathrm{Se}_{3}$ and $\mathrm{Bi}_{2} \mathrm{Te}_{3}$ with Highly Tunable Chemical Potential," Nano Lett., 10 [6] 2245-50 (2010).

46. J. Zheng, X. Yan, Z. Lu, H. Qiu, G. Xu, X. Zhou, P. Wang, X. Pan, K. Liu, and L. Jiao, "High-Mobility Multilayered $\mathrm{MoS}_{2}$ Flakes with Low Contact Resistance Grown by Chemical Vapor Deposition," Adv. Mater., 29 [13] 1604540 (2017).

47. S. M. Shinde, K. P. Dhakal, X. Chen, W. S. Yun, J. Lee, H. Kim, and J.-H. Ahn, "Stacking-Controllable Interlayer Coupling and Symmetric Configuration of Multilayered $\mathrm{MoS}_{2}, " N P G$ Asia Mater., 10 e468 (2018).

48. J. C. Park, S. J. Yun, H. Kim, J.-H. Park, S. H. Chae, S.-J. An, J.-G. Kim, S. M. Kim, K. K. Kim, and Y. H. Lee, "Phase-Engineered Synthesis of Centimeter-Scale $1 \mathrm{~T}^{\prime}$ and 2H-Molybdenum Ditelluride Thin Films," ACS Nano, 9 [6] 6548-54 (2015).

49. S. Cho, S. Kim, J. H. Kim, J. Zhao, J. Seok, D. H. Keum, J. Baik, D.-H. Choe, K. J. Chang, and K. Suenaga, "Phase Patterning for Ohmic Homojunction Contact in $\mathrm{MoTe}_{2}$," Science, 349 [6248] 625-28 (2015).

50. S. Song, D. H. Keum, S. Cho, D. Perello, Y. Kim, and Y. H. Lee, "Room Temperature Semiconductor-Metal Transition of $\mathrm{MoTe}_{2}$ Thin Films Engineered by Strain," Nano Lett., 16 [1] 188-93 (2015).

51. Y. Li, K.-A. N. Duerloo, K. Wauson, and E. J. Reed, "Structural Semiconductor-to-Semimetal Phase Transition in Two-Dimensional Materials Induced by Electrostatic Gating," Nat. Commun., 710671 (2016).

52. J. H. Sung, H. Heo, S. Si, Y. H. Kim, H. R. Noh, K. Song, J. Kim, C.-S. Lee, S.-Y. Seo, and D.-H. Kim, "Coplanar Semiconductor-Metal Circuitry Defined on Few-Layer $\mathrm{MoTe}_{2}$ via Polymorphic Heteroepitaxy," Nat. Nanotechnol., 12 1064-70 (2017).

53. T. A. Empante, Y. Zhou, V. Klee, A. E. Nguyen, I.-H. Lu, M. D. Valentin, S. A. Naghibi Alvillar, E. Preciado, A. J. Berges, and C. S. Merida, "Chemical Vapor Deposition Growth of Few-Layer $\mathrm{MoTe}_{2}$ in the 2H, 1T', and 1T Phases: Tunable Properties of $\mathrm{MoTe}_{2}$ Films," ACS Nano, 11 [1] 900-5 (2017). 